doi:10.1017/\$1041610221000880

\section{Disseminating palliative care education to Chinese Americans through social media}

The elderly population often suffers from multiple complex chronic illnesses. Hence, initiating timely discussions regarding advance care planning (ACP) and palliative care should be essential. For elderly Chinese American immigrants, due to low acculturation, they often suffer from limited knowledge and engagement in ACP (Dhingra et al., 2020). Lack of knowledge has been cited as the main reason for non-completion of advance directives or ACP (Yonashiro-Cho et al., 2016).

Disseminating information in the appropriate language is likely a crucial factor. According to the Pew Research Center analysis of 2013-2015 American Community Survey, over $40 \%$ of Chinese population in the USA $\geq 5$ years of age were not English proficient (Pew Research Center, 2017). Thus, the language barrier may be preventing Chinese Americans from understanding and engaging in ACP. Our study examines the viewing and distribution habits among the Chinese-speaking public through various social media platforms regarding a palliative care education video.

A board-certified psychiatrist was invited onto a Chinese radio broadcast station for an educational talk show in Cantonese, discussing the nature, purpose, and benefits of palliative care. The real-time recording was then uploaded to YouTube, as a singular 46.91-minute video (https://youtu.be/2kAsTvAY8_Y). Using YouTube Analytics, the data extrapolated included parameters such as type of device used, age of viewers, number of views, average view duration (AVD), and number of times the video was shared, further categorized by each social media platform.

From January 2014 to January 2019, data captured from the YouTube video showed 1,594 views, all by viewers aged 55 years and older for a total watch time of 7,416 minutes. The overall AVD was 4.65 minutes $(9.91 \%$ of entire video). Viewers watched the content using mobile phone $(41.72 \%$, $n=665)$, computer $(41.84 \%, n=667)$, or tablet $(16.44 \%, n=262)$. The AVD for mobile phone is 5.25 minutes, for computer is 4.01 minutes, and for tablet is 4.60 minutes. Of the traffic sources, 69 views $(4.32 \%)$ were externally sourced, with communication platforms being Google Search $(n=27)$,
WhatsApp $(n=11)$, Facebook $(n=8)$, and others $(n=23)$. The AVD of Google Search is $2.15 \mathrm{~min}-$ utes, of WhatsApp is 0.54 minutes, and of Facebook is 4.01 minutes. The video was shared 19 times with $73.68 \%(n=14)$ through WhatsApp, $15.79 \%(n=3)$ through text message, and $10.53 \%(n=2)$ through email.

To our knowledge, there are no reports of utilizing social media to disseminate palliative care knowledge to specifically Chinese-speaking Americans. Previous studies have demonstrated the feasibility of YouTube videos in disseminating health information to the Chinese-speaking general public (Lam et al., 2017; Shu and Woo, 2017). The current study adds that wireless devices maybe be the method of choice to disseminate information across various social media platforms. Majority (58.16\%) of viewers utilized wireless devices, with mobile phones achieving the highest AVD among all the devices used. Based on this study, future research should explore how to increase watch time of language-appropriate palliative care videos on mobile phones and how to increase reach and visibility of similar videos on social media platforms.

\section{Conflict of interest}

The authors have no financial, personal, or other conflicts of interest.

\section{Description of authors' roles}

W. Kuang designed the study, carried out the statistical analysis, and wrote the paper. B. Woo was responsible for collecting and providing the data, and assisted with writing the paper.

\section{References}

Dhingra, L. et al. (2020). Attitudes and beliefs toward advance care planning among underserved ChineseAmerican immigrants. Fournal of Pain and Symptom Management, 60(3), 588-594. https://doi.org/10.1016/j .jpainsymman.2020.04.013

Lam, N. H. T., Tsiang, J. T. H. and Woo, B. K. P. (2017). Exploring the role of YouTube in disseminating psychoeducation. Academic Psychiatry, 41(6), 819-822. https://doi.org/10.1007/s40596-017-0835-9

Pew Research Center. (2017, July 6). English proficiency of Chinese populations in the U.S., 2015. Pew Research Center: 
Social \& Demographic Trends. https://www.pewresearch .org/social-trends/chart/english-proficiency-of-chinesepopulation-in-the-u-s/.

Shu, S. and Woo, B. K. P. (2020). Digital media as a proponent for healthy aging in the older Chinese American population: Longitudinal analysis. FMIR Aging, 3(1), e20321. https://doi.org/10.2196/20321

Yonashiro-Cho, J., Cote, S., and Enguidanos, S. (2016). Knowledge about and perceptions of advance care planning and communication of Chinese-American older adults.
Fournal of the American Geriatrics Society, 64(9), 1884-1889. https://doi.org/10.1111/jgs.14261

Wayne Kuang ${ }^{1}$ and Benjamin K.P. Woo ${ }^{2}$

${ }^{1}$ College of Osteopathic Medicine of the Pacific, Western University of Health Sciences, Pomona, CA, USA ${ }^{2}$ Department of Psychiatry \& Biobehavioral Science, University of California, Los Angeles, CA, USA E-mail: wayne.kuang@westernu.edu 